\title{
Síndrome lupus-like induzida pelo uso do infliximabe em paciente portador de doença inflamatória intestinal
}

\section{Lupus-like syndrome induced by the use of Infliximabe in a patient with inflammatory bowel disease}

Livia Maria Sampaio Pinheiro1. Lucia Libanez Bessa Campelo Braga ${ }^{1,2}$. Ranna Caroline Bezerra Siebra ${ }^{1,2,3}$. Isabele de Sá Silveira Melo ${ }^{1,2,3}$. Sarah Araujo Loss ${ }^{1}$.

1 Universidade Federal do Ceará (UFC), Fortaleza, Ceará, Brasil. 2 Hospital Universitário Walter Cantídio (HUWC), Ceará, Brasil. 3 Empresa Brasileira de Serviços Hospitalares (EBSERH), Fortaleza, Ceará, Brasil.

\section{RESUMO}

As doenças inflamatórias intestinais (DII) são afecções imunomediadas do trato digestivo cujo tratamento pode incluir os antiTNF alfa. Sabe-se que essas drogas podem causar desordens autoimunes, que variam desde a indução de auto anticorpos a doenças autoimunes mais sérias, como a síndrome lupus-like, que é incomum e pouco descrita na literatura. Nesse estudo, é apresentado um caso de paciente do sexo feminino, 29 anos, portadora de Doença de Crohn fistulizante, que, após 1 ano e 5 meses do uso do infliximabe evoluiu com quadro de artrite periférica em articulações das mãos, punhos e joelhos e, após 5 meses, rash malar e fotossensibilidade. A dosagem do FAN foi 1:640, padrão homogêneo, e C4 estava reduzido. Nesse contexto, foi suspeitado da síndrome lupus-like induzida pelo uso do anti-TNF alfa, sendo iniciado prednisona, e o infliximabe foi substituído por adalimumabe, ocorrendo resolução dos sintomas. Não há critérios diagnósticos específicos para lúpus induzido por drogas e geralmente os pacientes apresentam sintomas cutâneo-articulares e auto anticorpos positivos, ocorrendo melhora clínica após suspensão da droga. É controverso se deveria ser reiniciado outro anti-TNF alfa ou um biológico de outra classe, sendo necessário novos estudos sobre a segurança dessas drogas em pacientes que desenvolveram a síndrome lupus-like.

Palavras-chave: Infliximab. Efeitos adversos. Doença inflamatória intestinal.

\section{ABSTRACT}

Inflammatory bowel diseases (IBD) are immune-mediated disorders of the digestive tract whose treatment may include antiTNF alpha. It is known that these drugs can cause autoimmune disorders, ranging from the induction of autoantibodies to more serious autoimmune diseases, such as the lupus-like syndrome, which is uncommon and poorly described in the literature. In this study, a case of 29-year-old female patient with Fistulizing Crohn's Disease was presented, who after 1 year and 5 months of use of infliximab developed peripheral arthritis in joints of the hands, wrists and knees and, after 5 months, malar rash and photosensitivity. The ANA dosage was 1: 640, homogeneous pattern and C4 was reduced. In this context, the lupus-like syndrome induced by the use of anti-TNF alpha was suspected, and prednisone was started, and infliximab was replaced by adalimumab. After that, the symptoms resolved. There are no specific diagnostic criteria for drug-induced lupus and patients usually develop cutaneous-articular symptoms and autoantibodies positive, with clinical improvement after drug withdrawal. It is controversial whether another anti-TNF alpha or a biological of another class should be restarted, and further studies on the safety of these drugs are needed in patients who have developed lupus-like syndrome.

Keywords: Infliximab. Adverse effects. Inflammatory bowel disease.

Autor correspondente: Livia Maria Sampaio Pinheiro, Rua Coronel Nunes de Melo, 1315, Rodolfo Teófilo, Fortaleza, Ceará, Brasil. CEP: 60430270. Telefone: +55 85 99634-3223. E-mail: liviamsp@hotmail.com

Conflito de interesses: Não há qualquer conflito de interesses por parte de qualquer um dos autores.

Recebido em: 08 Jul 2019; Revisado em: 26 Out 2019; Aceito em: 14 Nov 2019. 


\section{INTRODUÇÃO}

As doenças inflamatórias intestinais (DII), que incluem principalmente a Doença de Crohn e a Retocolite Ulcerativa Idiopática, são afecções crônicas imunomediadas do trato gastrointestinal cuja etiologia envolve uma interação complexa entre fatores genéticos, microbiota intestinal e imunorregulação de mucosa. ${ }^{1-3}$

No geral, a RCUI apresenta processo inflamatório localizado na mucosa colônica e retal, de forma contínua, enquanto a DC pode afetar qualquer localização do trato gastrointestinal, da boca ao ânus, com propensão a região distal do íleo e proximal do colon, de forma salteada e com acometimento transmural, ${ }^{1,2}$ podendo complicar com fistulas, estenoses e abscessos. ${ }^{2}$

Para combater esse processo inflamatório, foram desenvolvidas drogas que bloqueiam a ação do fator de necrose tumoral alfa (TNF-alfa), uma citocina pro-inflamatória envolvida na patogênese de várias doenças autoimunes, incluindo as DII. ${ }^{4}$ Essas drogas, que incluem Infliximabe, Etanecerpt, Adalimumabe e, mais recentemente, certolizumabe e golimumabe, têm sido um importante avanço no tratamento das DII e vêm sendo cada vez mais utilizadas em pacientes com doença moderada a severa e nos pacientes refratários ao tratamento convencional, podendo ser ainda terapia de primeira linha em alguns casos. ${ }^{5}$ Contudo, ao bloquearem os efeitos fisiológicos dessa citocina, os antagonistas do TNF-alfa podem levar a efeitos adversos, como neoplasias, infecções, e desordens auto imunes. ${ }^{6}$

As desordens autoimunes variam de alterações imunológicas assintomáticas a doenças autoimunes mais sérias, como a síndrome lupus-like. Sabe-se que frequentemente os antiTNF-alfa induzem a produção de auto anticorpos, ${ }^{7-9}$ mas o surgimento da síndrome lupus-like é incomum, com uma prevalência de 0,5 a $1 \%$ nos pacientes em uso dessas drogas. ${ }^{10}$ Os indivíduos afetados geralmente referem sintomas cutâneoarticulares, sendo raro o envolvimento de órgãos sólidos, e a melhora ocorre após suspensão do uso da droga. ${ }^{1,9}$

Pelo crescente número de pacientes em uso dos anti-TNF-alfa na DII, é necessário conhecer os efeitos adversos relacionados a essas drogas, especialmente os mais raros, como a síndrome lupus-like, que é pouco descrita na literatura.

\section{RELATO DO CASO}

Paciente do sexo feminino, 29 anos, diagnosticada em 2010 com Doença de Crohn, fez uso inicialmente de prednisona e sulfassalazina, deixando a paciente assintomática até 2014. Em janeiro de 2015, após apendicectomia por quadro agudo de apendicite, a paciente evoluiu com fístula enterocutânea na fossa ilíaca direita, sendo iniciado azatioprina e infliximabe. Houve boa evolução do quadro, com fechamento da fístula cerca de 30 dias após início do imunobiológico. Após 1 ano e 5 meses de uso assintomático do infliximabe, em março de 2017, a paciente iniciou quadro de artrite periférica nãoerosiva em interfalangianas proximais, metatarsofalangianas, punhos e joelhos, sem deformidade articular. Após 5 meses, evoluiu com rash malar e fotossensibilidade. Em seguida, foi solicitado dosagem do fator anti-nuclear (FAN), com resultado de 1:640 padrão homogêneo, e $\mathrm{C} 4$, que estava reduzido, com valor de $16 \mathrm{mg} / \mathrm{dL}$ (Valor de referência $>19 \mathrm{mg} / \mathrm{dL}$ ). O hemograma, C3, CH-50, anti-RNP, anti-Sm, anti-DNA, anti-histona, anti-Ro, anti-La e HLAB27 não evidenciaram alterações. Também não houve alteração na função renal, hepática, neurológica e cardiopulmonar. Ao correlacionar o quadro clínico e laboratorial no contexto do uso do infliximabe, foi suspeitado da síndrome lupus-like induzida pelo uso do anti-TNF-alfa. Em seguida, foi iniciado prednisona $20 \mathrm{mg}$ por dia e o infliximabe foi substituído por adalimumabe, ocorrendo completa resolução do quadro clínico em 1 mês. A prednisona foi utilizada por curto período, com desmame completo em 2 meses. Durante o seguimento, houve negativação do FAN, normalização do $\mathrm{C} 4$ e não houve recorrência dos sintomas relacionados ao lúpus em 6 meses após troca de imunobiológico. Em relação a doença de Crohn, a paciente continuou em remissão com o uso do adalimumabe e azatioprina.

\section{DISCUSSÃO}

O Infliximabe tem sido bastante estudado na última década, demonstrando ser uma droga com boa segurança e tolerabilidade. Contudo, sérios e inesperados eventos adversos têm sido descritos, como a síndrome lupus-like. ${ }^{9}$

A síndrome lupus-like por uso de anti-TNF é rara, ocorre mais comumente em portadores de artrite reumatoide do que na doença de Crohn e acomete mais frequentemente mulheres do que homens (2:1). ${ }^{11}$ Não há critérios bem definidos para diagnosticar o lúpus induzido por drogas e, muitas vezes, os pacientes nestes casos não satisfazem os critérios para Lúpus Eritematoso Sistêmico (LES) propostos pelo Colégio Americano de Reumatologia (CAR). Geralmente ocorre um ou mais sintomas compatíveis com LES no contexto do uso dessas drogas, ocorrendo resolução do quadro após suspensão do uso do anti-TNF alfa. ${ }^{8,9} \mathrm{Na}$ maioria das vezes o quadro é leve, com manifestações cutâneas, como rash malar e fotossensibilidade, e sintomas musculoesqueléticos, como artrite não erosiva, além da presença de auto anticorpos. ${ }^{9}$ É raro o envolvimento de outros órgãos, como rins, coração, fígado e pulmão. No caso relatado, a paciente apresentou 4 dos 11 critérios para LES idiopático do CAR (rash malar, fotossensibilidade, artrite periférica e a positividade do Fator antinuclear).

De Bandt $\mathrm{M}$ et al demonstrou que a positividade de anticorpos antinucleares variam de $79-100 \%$ e do Anti-DNA, de 72 a $92 \%,{ }^{7}$ e Costa $\mathrm{MF}$ et al evidenciou que a redução do complemento ocorre em mais de $50 \%$ dos pacientes com síndrome lúpus-like por uso do anti-TNF alfa. ${ }^{7-9}$ As citopenias são as alterações hematológicas mais comuns, ${ }^{12}$ embora não tenham sido demonstradas no caso relatado.

Os mecanismos fisiopatogênicos do desenvolvimento da síndrome ainda são incertos, mas existem várias teorias. 
A primeira hipótese seria que a supressão dos linfócitos Th1 causada pelo uso do anti-TNF alfa levaria ao aumento dos linfócitos Th2 e consequente produção de linfócitos B produtores de auto anticorpos. A segunda hipótese é baseada no aumento de infecções bacterianas pelo uso do biológico, o que levaria a ativação de linfócitos e formação de auto anticorpos. A terceira hipótese seria que o tratamento com anti-TNF alfa levaria à exposição de material nuclear de células apoptóticas, que agiriam como antígenos, e consequentemente ocorreria formação de auto anticorpos. , $^{5,12,13}$

O tratamento concomitante da doença de base com outro imunossupressor, como a azatioprina, parece ser fator protetor para o desenvolvimento da síndrome Lúpus-like, já que potencialmente ocorreria redução da taxa de produção de auto anticorpos, ${ }^{14,12}$ o que não ocorreu no caso relatado.

$\mathrm{Na}$ grande maioria dos casos ocorre resolução do quadro com a descontinuação do anti-TNF alfa, principalmente em pacientes com sintomas leves. Em alguns casos, pode haver necessidade do uso de corticoides e/ou imunossupressores, o que geralmente ocorre nos pacientes com sintomas mais intensos e/ou com acometimento neurológico, renal ou pulmonar. ${ }^{9,12}$ Ramos-Casals $\mathrm{M}$ et al sugere que é opcional a suspensão da droga em pacientes com sintomas leves ou somente com auto anticorpos positivos, devendo-se apenas fazer o acompanhamento do paciente de forma mais frequente. ${ }^{9}$

É controverso se deveria ser reiniciado um outro anti-TNF alfa ou um biológico de outra classe, já que há um risco potencial

\section{REFERÊNCIAS}

1. Mulder D, Noble A, Justinich C, Duffin J. A tale of two diseases: the history of inflammatory bowel disease. J Crohns Colitis. 2014;8(5):341-8.

2. Feldman M, Friedman LS, Brandt LJ, editors. Sleisenger and Fordtran's Gastrointestinal and Liver Disease. New York: Saunders Elsevier; 2010.

3. Jostins L, Ripke S, Weersma RK, Duerr RH, McGovern DP, Hui KY, et al. Host-microbe interactions have shaped the genetic architecture of inflammatory bowel disease. Nature. 2012;491(7422):119-24.

4. Bank S, Andersen PS, Burisch J, Pedersen N, Roug S, Galsgaard $\mathrm{J}$, et al. Associations between functional polymorphisms in the NFKB signaling pathway and response to anti-TNF treatment in Danish patients with inflammatory bowel disease. Pharmacogenomics J. 2014;14(6):526-34.

5. Verma HD, Scherl EJ, Jacob VE, Bosworth BP. Anti-nuclear antibody positivity and the use of certolizumab in inflammatory bowel disease patients who have had arthralgias or lupus-like reactions from infliximab or adalimumab. J Dig Dis. 2011;12(5):37983.

6. Borras-Blasco J, Navarro-Ruiz A, Borras C, Castera E. Adverse cutaneous reactions induced by TNF alpha antagonist therapy. South Med J. 2009;102(11):1133-40. de ressurgimento do quadro com o uso de outro anti-TNF alfa. A maioria dos estudos mostram que a substituição entre os antagonistas do TNF alfa é segura. Wetter DA et al, em estudo feito na Mayo Clinic, demonstrou que 4 de 5 pacientes com a síndrome lupus-like que fizeram uso de outro anti-TNF alfa apresentaram boa evolução do quadro e controle da doença de base, sem recorrência dos sintomas relacionados ao lúpus. ${ }^{15}$ Verma HD mostrou que 5 de 6 pacientes que apresentaram a síndrome após uso de infliximabe ou adalimumabe não tiveram recorrência dos sintomas após substituição por certolizumabe. ${ }^{5}$ Acredita-se que o certolizumabe apresenta baixas taxas de produção de autoanticorpos e reação lupuslike em relação aos outros anti-TNF alfa devido a sua estrutura única e, desse modo, seria uma opção mais segura nos casos que evoluem com desordens autoimunes. ${ }^{5}$

No relato de caso descrito não houve recorrência dos sintomas relacionados ao lúpus em 6 meses após substituição do infliximabe pelo adalimumabe e a paciente permaneceu em remissão da doença de base.

Dessa forma, é importante que pacientes em uso de antiTNF alfa tenham seguimento regular, sendo mandatório a investigação de desordem imunológica se ocorrer surgimento de quadro cutâneo-articular. Geralmente, o tratamento consiste na suspensão da droga e sua substituição por outro imunobiológico. São necessários novos estudos sobre a segurança de agentes anti-TNF alfa alternativos em pacientes que desenvolveram a síndrome lúpus-like ou outras desordens autoimunes.

7. De Bandt M, Sibilia J, Le Loët X, Prouzeau S, Fautrel B, Marcelli $\mathrm{C}$, et al. Systemic lupus erythematosus induced by anti-tumour necrosis factor alpha therapy: a French national survey. Arthritis Res Ther. 2005;7(3):545-51.

8. Costa MF, Said NR, Zimmermann B. Drug-induced lúpus due to anti-tumor necrosis factor alpha agents. Semin Arthritis Rheum. 2008;37(6):381-7.

9. Ramos-Casals M, Brito-Zerón P, Muñoz S, Soria N, Galiana D, Bertolaccini L, et al. Autoimmune diseases induced by TNFtargeted therapies: analysis of 233 cases. Medicine (Baltimore). 2007;86(4):242-51.

10. Aringer M, Smolen JS. The role of tumor necrosis factor-alpha in systemic lupus erythematosus. Arthritis Res Ther. 2008;10(1):202.

11. Moulis G, Sommet A, Lapeyre-Mestre M, Montastruc JL; Association Française des Centres Régionaux de PharmacoVigilance. Is the risk of tumour necrosis factor inhibitorinduced lupus or lupus-like syndrome the same with monoclonal antibodies and soluble receptor? A case/non-case study in a nationwide pharmacovigilance database. Rheumatology (Oxford). 2014;53(10):1864-71.

12. Lupu A, Tieranu C, Constantinescu CL, Diculescu M. TNF $\alpha$ inhibitor induced lupus-like syndrome (TAILS) in a patient with IBD. Curr Health Sci J. 2014;40(4):285-8. 
13. Via CS, Shustov A, Rus V, Lang T, Nguyen P, Finkelman FD. In vivo neutralization of TNF-alpha promotes humoral autoimmunity by preventing the induction of CTL. J Immunol. 2001;167(12):6821-6.

14. Beigel F, Schnitzler F, Paul Laubender R, Pfennig S, Weidinger M, Göke B, et al. Formation of antinuclear and double-strand DNA antibodies and frequency of lupus-like syndrome in anti-TNF-alfa. Inflamm Bowel Dis. 2011;17(1):91-8.

15. Wetter DA, Davis MD. Lupus-like syndrome attributable to antitumor necrosis factor alpha therapy in 14 patients during an 8-year period at Mayo Clinic. Mayo Clinic Proc. 2009;84(11):979-84.

\section{Como citar:}

Pinheiro LM, Braga LL, Siebra RC, Melo IS, Loss SA. Síndrome lupus-like induzida pelo uso do infliximabe em paciente portador de doença inflamatória intestinal. 2020 abr-jun;60(2):47-50. 